\title{
Unconditionally Stable Pressure-Correction Schemes for a Linear Fluid-Structure Interaction Problem
}

\author{
Ying He and Jie Shen* \\ Department of Mathematics, Purdue University, West Lafayette, IN 47907, USA
}

\begin{abstract}
We consider in this paper numerical approximation of the linear fluid-structure interaction (FSI). We construct a new class of pressure-correction schemes for the linear FSI problem with a fixed interface, and prove rigorously that they are unconditionally stable. These schemes are computationally very efficient, as they lead to, at each time step, a coupled linear elliptic system for the velocity and displacement in the whole region and a discrete Poisson equation in the fluid region.
\end{abstract}

AMS subject classifications: 74F10, 76D05, 65M12, 35Q30

Key words: Fluid-structure interaction, pressure-correction, stability analysis.

\section{Introduction}

Fluid-Structure Interaction (FSI) plays an important role in many scientific/engineering applications, e.g., design of engineering systems, blood flow in human arteries, etc. It has been extensively studied in recent years both analytically and computationally (cf. $[5,7,9,14]$ and the references therein).

The fluid velocity, pressure and structure displacement in the FSI problems are coupled together, making it difficult to solve numerically. For fluid problems, an effective approach to decouple the computation of the pressure from that of the velocity is to use a so-called projection type method, originally proposed by Chorin and Temam in the late 60 's. A comprehensive review on various projection type methods can be found in [12]. However, a main difficulty in the design of a projection method is what boundary condition to use for the pressure at the interface. It is well known that a proper boundary condition, at the Dirichlet part of the boundary, for the pressure Poisson equation in a projection type method is the homogeneous Neumann boundary condition. Indeed, most existing projection type schemes (cf., for instance, $[1,3,10]$ ) for FSI problem also use, explicitly or implicitly, Neumann type boundary condition for the pressure Poisson equation at the Dirichlet part of the boundary as well as at the interface. However, imposing a Neumann type boundary condition for the pressure at the interface appears to affect, in certain

${ }^{*}$ Corresponding author. Email addresses: he14@math.purdue.edu (Ying He), shen@math.purdue.edu (Jie Shen)

http://www.global-sci.org/nmtma 
degree, the stability of the scheme, and we are not aware of any proof of unconditional stability for this type of projection scheme for the FSI problem, although a conditional stability has been proven in [10].

In [11], the authors proposed and analyzed pressure-correction projection schemes for Navier-Stokes equations with open boundary where the usual stress-free boundary condition is applied. It is shown that the proper boundary condition at the open boundary is of Dirichlet type instead of Neumann type. Two schemes are constructed in [11]. One is based on the standard pressure-correction which leads to poor accuracy at the open boundary, the other is based on the rotational pressure-correction and with a proper Dirichlet boundary condition at the open boundary. It is shown in [11] that both the standard and rotational pressure-correction projection schemes, when applied to the time-dependent Stokes problem, are unconditionally stable, but the rotational version leads to much better accuracy. Since one of matching interface condition for the FSI problem is related to the stress, it is sensible to extend the approach in [11] for problems with open boundary to the FSI problem.

In this paper, we shall construct a different class of projection semi-implicit schemes which decouple the computation of pressure from that of the velocity and structure displacement. Our schemes will be computationally very efficient. More precisely, in the first step of our schemes, we solve a coupled, but elliptic, system for an intermediate fluid velocity and the structure displacement, then in the second step, we solve a Poisson equation for the fluid pressure and obtain the fluid velocity with a simple correction. Furthermore, we shall also prove rigorously that these schemes are unconditionally stable.

To fix the idea, we consider in this paper a simple model of the FSI problem where the movement of the interface is assumed infinitesimal so the interface is treated as fixed. This linear FSI problem captures many of the essential difficulties of the more general FSI problems with moving interface.

The rest of the paper is organized as follows. In the next section, we describe the governing equations for our FSI model, formulate its weak form and the energy dissipation law. In Section 3, we construct standard and rotational pressure-correction scheme for the FSI problem, and prove their unconditional stability. Then, in Section 4, we describe a Fourier-Legendre method for a special case when the domain is a periodic channel. We present some numerical results in Section 5 to validate our numerical schemes and to demonstrate their temporal accuracy. Some concluding remarks are given in Section 6 .

\section{Governing Equations}

We consider the following model for interaction of a viscous fluid with an elastic body in a two- or three-dimensional bounded domain $\Omega$, with the fluid region $\Omega_{f}$, the solid region $\Omega_{s}$ and the interface $\Gamma_{c}$, so we have $\Omega=\Omega_{f} \cup \Omega_{s} \cup \Gamma_{c}$. We also denote $\Gamma_{f}=\partial \Omega_{f} \backslash \Gamma_{c}$ and $\Gamma_{s}=\partial \Omega_{s} \backslash \Gamma_{c}$ (cf. Fig. 1).

We assume that the interface undergoes infinitesimal displacements, i.e., $\Gamma_{c}$ is assumed

to be fixed. The more complicated situation with moving interface will be considered in a forthcoming paper. 


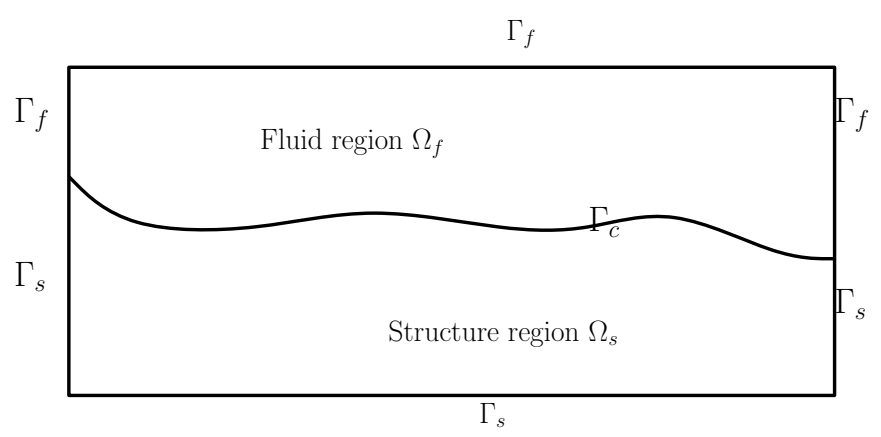

Figure 1: Geometry discription for fluid-structure problem

In the fluid region $\Omega_{f}$, we have the Stokes equations:

$$
\begin{array}{ll}
\rho_{f} u_{t}-\operatorname{div} \epsilon(u)+\nabla p=\rho_{f} f_{1}, & \text { in } \Omega_{f} \times(0, T), \\
\operatorname{div} u=0, & \text { in } \Omega_{f} \times(0, T), \\
u=0, & \text { on } \Gamma_{f} \times(0, T), \\
\left.u\right|_{t=0}=u_{0}, & \text { in } \Omega_{f}
\end{array}
$$

where $u$ denotes the fluid velocity, $p$ the fluid pressure, $u_{0}$ the given initial velocity, $f_{1}$ the given body force per unit mass, $\epsilon(u)=\frac{\mu}{2}\left(\nabla u+\nabla u^{T}\right)$ the strain tensor, $\rho_{f}$ and $\mu$ the constant fluid density and viscosity.

In the solid region $\Omega_{s}$, we have the wave equation for linear elasticity:

$$
\begin{array}{ll}
\rho_{s} w_{t t}-\operatorname{div} \sigma(w)=\rho_{s} f_{2}, & \text { in } \Omega_{s} \times(0, T), \\
w=0, & \text { on } \Gamma_{s} \times(0, T), \\
w(\cdot, 0)=w_{0}, & \text { in } \Omega_{s}, \\
w_{t}(\cdot, 0)=w_{1}, & \text { in } \Omega_{s}
\end{array}
$$

where $w$ denotes the displacement of the solid, $w_{0}$ and $w_{1}$ the given initial data, and $\sigma(w)$ the elastic stress tensor, given by

$$
\sigma_{i j}(w)=\lambda \sum_{k=1}^{3} \epsilon_{k k}(w)+2 \mu_{2} \epsilon_{i j}(w),
$$

$f_{2}$ the given loading force per unit mass, $\lambda$ and $\mu_{2}$ the Lamé constants, and $\rho_{s}$ the constant solid density.

Across the fixed interface $\Gamma_{c}$ between the fluid and solid, the velocity and the stress vector are required to be continuous, i.e.,

$$
w_{t}=u, \quad \text { on } \Gamma_{c} \times(0, T)
$$

and

$$
\sigma(w) \cdot \mathbf{n}=\epsilon(u) \cdot \mathbf{n}-p \mathbf{n}, \quad \text { on } \Gamma_{c} \times(0, T)
$$


where $\mathbf{n}$ denotes the outward normal vector along $\Gamma_{c}$ w.r.t. $\Omega_{s}$.

For simplicity, we take in this paper $\rho_{f}=\rho_{s}=1, f_{1}=f_{2}=0$. We further take $\lambda=1$ and $\mu_{2}=0$ which imply $\operatorname{div} \sigma(w)=\Delta w$, and the interface condition (2.4) is reduced to

$$
\frac{\partial w}{\partial \mathbf{n}}=\mu \frac{\partial u}{\partial \mathbf{n}}-p \mathbf{n}, \quad \text { on } \Gamma_{c} \times(0, T) .
$$

In order to derive a weak formulation for (2.1)- (2.2), we need to introduce some notations. Let us denote by $H^{k}(\Omega)$ and $H_{0}^{k}(\Omega)$ (for $k \geq 0$ ) the standard Sobolev spaces, equipped with the standard norm $\|\cdot\|_{k, \Omega}$. In particular, we denote $L^{2}(\Omega)=H^{0}(\Omega)$ with the associated norm $\|\cdot\|$. We will use $\mathbf{H}^{\mathrm{k}}\left(\Omega_{f}\right)$ to denote the vector-valued Sobolev spaces. We also denote

$$
H_{0, \Gamma_{f}}^{1}\left(\Omega_{f}\right)=\left\{v \in H^{1}\left(\Omega_{f}\right):\left.v\right|_{\Gamma_{f}}=0\right\}, \quad H_{0, \Gamma_{s}}^{1}\left(\Omega_{s}\right)=\left\{v \in H^{1}\left(\Omega_{s}\right):\left.v\right|_{\Gamma_{s}}=0\right\} .
$$

Then, a weak solution $(u, p, w)$ for (2.1)-(2.2) will satisfy

$$
\begin{aligned}
\left(u_{t}, \varphi\right)_{\Omega_{f}}+(\mu \nabla u, \nabla \varphi)_{\Omega_{f}}-(p, \operatorname{div} \varphi)_{\Omega_{f}}+\left(\mu \frac{\partial u}{\partial \mathbf{n}}-p \mathbf{n}, \varphi\right)_{\Gamma_{c}} & =0, \quad \forall \varphi \in \mathbf{H}_{0, \Gamma_{f}}^{1}\left(\Omega_{f}\right), \\
(\operatorname{div} u, q)_{\Omega_{f}} & =0, \quad \forall q \in L^{2}\left(\Omega_{f}\right), \\
\left(w_{t t}, \psi\right)_{\Omega_{s}}+(\nabla w, \nabla \psi)_{\Omega_{s}}-\left(\frac{\partial w}{\partial \mathbf{n}}, \psi\right)_{\Gamma_{c}} & =0, \quad \forall \psi \in \mathbf{H}_{0, \Gamma_{s}}^{1}\left(\Omega_{s}\right)
\end{aligned}
$$

with the interface conditions (2.3) and (2.5) on $\Gamma_{c}$.

We can reformulate the above, using (2.5), to:

$$
\begin{array}{rr}
\left(u_{t}+(u \cdot \nabla) u, \varphi\right)_{\Omega_{f}}+(\mu \nabla u, \nabla \varphi)_{\Omega_{f}}-(p, \operatorname{div} \varphi)_{\Omega_{f}}+\left(\frac{\partial w}{\partial \mathbf{n}}, \varphi\right)_{\Gamma_{c}}=0, & \forall \varphi \in \mathbf{H}_{0, \Gamma_{f}}^{1}\left(\Omega_{f}\right), \\
(\operatorname{div} u, q)_{\Omega_{f}}=0, & \forall q \in L^{2}\left(\Omega_{f}\right), \\
\left(w_{t t}, \psi\right)_{\Omega_{s}}+(\nabla w, \nabla \psi)_{\Omega_{s}}-\left(\frac{\partial w}{\partial n}, \psi\right)_{\Gamma_{c}}=0, & \forall \psi \in \mathbf{H}_{0, \Gamma_{s}}^{1}\left(\Omega_{s}\right)
\end{array}
$$

with $u=w_{t}$ on the interface $\Gamma_{c}$.

Setting $\varphi=u, \psi=w_{t}$ in (2.7a) and (2.7c), and summing up the two resultant equations, we obtain

$$
\frac{1}{2} \partial_{t}\|u\|_{\Omega_{f}}^{2}+\mu\|\nabla u\|_{\Omega_{f}}^{2}+\frac{1}{2} \partial_{t}\left\|w_{t}\right\|_{\Omega_{f}}^{2}+\frac{1}{2} \partial_{t}\|\nabla w\|_{\Omega_{s}}^{2}=0
$$

or equivalently

$$
\partial_{t}\left\{\|u\|_{\Omega_{f}}^{2}+\left\|w_{t}\right\|_{\Omega_{f}}^{2}+\|\nabla w\|_{\Omega_{s}}^{2}\right\}=-2 \mu\|\nabla u\|_{\Omega_{f}}^{2} \leq 0
$$


where

$$
E\left(u, w, w_{t}\right):=\|u\|_{\Omega_{f}}^{2}+\left\|w_{t}\right\|_{\Omega_{f}}^{2}+\|\nabla w\|_{\Omega_{s}}^{2}
$$

is the total energy of the FSI system.

Thus, the well posedness of the system (2.7) can be easily established by using a standard procedure. In fact, the well posedness of a related nonlinear system, with the Stokes equations (2.1) replaced by the Navier-Stokes equations, is studied in [17].

In [6], the authors studied a semi-discrete (in space) finite-element method for the linear FSI problem with a fixed interface. On the other hand, we shall be mainly concerned with semi-discrete (in time) projection type schemes.

\section{Time Discretization}

For FSI problems, it is very important to design numerical schemes which have good stability property. Usually, this is achieved by fully coupled, implicit schemes which require solving, at each time step, a coupled saddle-point system.

We construct in this section time discretization schemes based on standard and rotational pressure-correction approach for (2.7). These schemes are unconditionally stable, and lead to, at each time step, a coupled, linear elliptic system in $\Omega$ and a pressure Poisson equation in $\Omega_{f}$, which can be efficiently solved by standard numerical methods. The stability analysis for each scheme is carried out in this section.

\subsection{Standard Pressure-Correction Scheme}

We first construct a first-order scheme for the FSI problem based on the standard pressure-correction scheme for Navies-Stokes problem with open boundary condition [11]:

Step 1 : Given $\left(u^{n}, p^{n}, w^{n}\right)$, compute $\tilde{u}^{n+1} \in \mathbf{H}_{0, \Gamma_{f}}^{1}\left(\Omega_{f}\right)$ and $w^{n+1} \in \mathbf{H}_{0, \Gamma_{s}}^{1}\left(\Omega_{s}\right)$ by solving

$$
\begin{aligned}
\left(\frac{\tilde{u}^{n+1}-u^{n}}{\Delta t}, \varphi\right)_{\Omega_{f}}+\left(\mu \nabla \tilde{u}^{n+1}, \nabla \varphi\right)_{\Omega_{f}}-\left(p^{n}, \operatorname{div} \varphi\right)_{\Omega_{f}}+\left(\frac{\partial w^{n+1}}{\partial \mathbf{n}}, \varphi\right)_{\Gamma_{c}} & =0 \\
\tilde{u}^{n+1}=\frac{w^{n+1}-w^{n}}{\Delta t}, & \text { on } \quad \Gamma_{c} \\
\left(\frac{w^{n+1}-2 w^{n}+w^{n-1}}{\Delta t^{2}}, \psi\right)_{\Omega_{s}}+\left(\nabla w^{n+1}, \nabla \psi\right)_{\Omega_{s}}-\left(\frac{\partial w^{n+1}}{\partial \mathbf{n}}, \psi\right)_{\Gamma_{c}} & =0
\end{aligned}
$$

for all $\varphi \in \mathbf{H}_{0, \Gamma_{f}}^{1}\left(\Omega_{f}\right)$ and $\psi \in \mathbf{H}_{0, \Gamma_{s}}^{1}\left(\Omega_{s}\right)$.

This is a coupled, linear elliptic system for $\left(\tilde{u}^{n+1}, w^{n+1}\right)$, with the coupling condition at the interface $\Gamma_{c}$. Hence, it can be efficiently solved, for example, by a standard domain decomposition approach (cf., for instance, $[20,22]$ ). 
Step 2 : Compute $u^{n+1} \in \mathbf{H}^{1}\left(\Omega_{f}\right)$ and $p^{n+1} \in H^{1}\left(\Omega_{f}\right)$ by solving

$$
\begin{aligned}
& \frac{u^{n+1}-\tilde{u}^{n+1}}{\Delta t}+\nabla\left(p^{n+1}-p^{n}\right)=0, \\
& \operatorname{div} u^{n+1}=0, \quad \text { in } \quad \Omega_{f}, \\
& \left.u^{n+1} \cdot \mathbf{n}\right|_{\Gamma_{f}}=0 \text { and }\left.p^{n+1}\right|_{\Gamma_{c}}=\left.p^{n}\right|_{\Gamma_{c}} .
\end{aligned}
$$

We observe that a Dirichlet boundary condition is imposed for $p^{n+1}$ on the interface $\Gamma_{c}$, as opposed to the usual Neumann boundary condition in a pressure-correction formulation. This is due to the interface condition (2.5) which is similar to the open boundary condition considered in [11].

We denote $H_{0, \Gamma_{c}}^{1}\left(\Omega_{f}\right)=\left\{q \in H^{1}\left(\Omega_{f}\right):\left.q\right|_{\Gamma_{c}}=0\right\}$. Then, the above system is equivalent to: Find $\left(p^{n+1}-p^{n}\right) \in H_{0, \Gamma_{c}}^{1}\left(\Omega_{f}\right)$ such that

$$
\begin{aligned}
\left(\nabla\left(p^{n+1}-p^{n}\right), \nabla q\right) & =-\frac{1}{\triangle t}\left(\nabla \cdot \tilde{u}^{n+1}, q\right), \quad \forall q \in H_{0, \Gamma_{c}}^{1}\left(\Omega_{f}\right), \\
u^{n+1} & =\tilde{u}^{n+1}-\Delta t \nabla\left(p^{n+1}-p^{n}\right) .
\end{aligned}
$$

Hence, we only have to solve a Poisson equation at this step.

For the above scheme, we have the following result:

Theorem 3.1. The scheme (3.1)-(3.3), with $\left.p^{0}\right|_{\Gamma_{c}}=0$, is unconditionally stable. More precisely, if we define the discrete energy

$$
E^{n}=\left\|u^{n}\right\|+\left\|\delta_{t} w^{n}\right\|^{2}+\left\|\nabla w^{n}\right\|^{2}+(\triangle t)^{2}\left\|\nabla p^{n}\right\|^{2}
$$

then we have, for all $n \geq 0$,

$$
E^{n+1}-E^{n}+\left\|\tilde{u}^{n+1}-u^{n}\right\|^{2}+2 \mu \triangle t\left\|\nabla \tilde{u}^{n+1}\right\|^{2}+\triangle t^{2}\left\|\delta_{t t}^{2} w^{n+1}\right\|^{2}+\triangle t^{2}\left\|\nabla\left(\delta_{t} w^{n+1}\right)\right\|^{2} \leq 0 .
$$

Proof. To simplify the notations, we define, for any sequence $\left\{u^{k}\right\}$, the discrete time derivatives $\delta_{t} u^{n+1}:=\frac{u^{n+1}-u^{n}}{\Delta t}$ and $\delta_{t t}^{2} u^{n+1}:=\frac{\delta_{t} u^{n+1}-\delta_{t} u^{n}}{\Delta t}=\frac{u^{n+1}-2 u^{n}+u^{n-1}}{\Delta t^{2}}$.

Taking $\varphi=2 \tilde{u}^{n+1}$ in (3.1a), $\psi=2 \delta_{t} w^{n+1}$ in (3.1c), and taking the inner product of (3.2a) with $q=2 \Delta t \nabla p^{n}$, then summing up the three relations, we obtain:

$$
\begin{aligned}
& \frac{1}{\Delta t}\left\{\left\|\tilde{u}^{n+1}\right\|^{2}-\left\|u^{n}\right\|^{2}+\left\|\tilde{u}^{n+1}-u^{n}\right\|^{2}\right\}+2\left\|\nabla \tilde{u}^{n+1}\right\|^{2}-2\left(p^{n}, \operatorname{div} \tilde{u}^{n+1}\right)_{\Omega_{f}} \\
& +\frac{1}{\Delta t}\left\{\left\|\delta_{t} w^{n+1}\right\|^{2}-\left\|\delta_{t} w^{n}\right\|^{2}+\left\|\delta_{t} w^{n+1}-\delta_{t} w^{n}\right\|^{2}\right\} \\
& +\frac{1}{\triangle t}\left\{\left\|\nabla w^{n+1}\right\|^{2}-\left\|\nabla w^{n}\right\|^{2}+\triangle t^{2}\left\|\nabla \delta_{t} w^{n+1}\right\|^{2}\right\}=0 .
\end{aligned}
$$

Rewrite (3.2a) as

$$
\frac{u^{n+1}}{\sqrt{\triangle t}}+\sqrt{\triangle t} \nabla p^{n+1}=\frac{\tilde{u}^{n+1}}{\sqrt{\triangle t}}+\sqrt{\triangle t} \nabla p^{n}
$$


Taking inner product with itself from both sides and integrating by parts, thanks to $\left.p^{k}\right|_{\Gamma_{c}}=$ 0 for all $k$ (due to $\left.p^{0}\right|_{\Gamma_{c}}=0$ ), and $\left.\tilde{u}^{n+1} \cdot \mathbf{n}\right|_{\Gamma_{f}}=0=\left.u^{n+1} \cdot \mathbf{n}\right|_{\Gamma_{f}}$, we obtain

$$
\frac{1}{\triangle t}\left\|u^{n+1}\right\|^{2}+\triangle t\left\|\nabla p^{n+1}\right\|^{2}=\frac{\left\|\tilde{u}^{n+1}\right\|^{2}}{\Delta t}+\triangle t\left\|\nabla p^{n}\right\|^{2}-2\left(p^{n}, \operatorname{div} \tilde{u}^{n+1}\right)_{\Omega_{f}} .
$$

Summing up (3.4) and (3.6), we obtain

$$
\begin{aligned}
& \frac{1}{\Delta t}\left\{\left\|u^{n+1}\right\|^{2}-\left\|u^{n}\right\|^{2}+\left\|\tilde{u}^{n+1}-u^{n}\right\|^{2}\right\}+2\left\|\nabla \tilde{u}^{n+1}\right\|^{2} \\
& +\frac{1}{\Delta t}\left\{\left\|\delta_{t} w^{n+1}\right\|^{2}-\left\|\delta_{t} w^{n}\right\|^{2}+\left\|\delta_{t} w^{n+1}-\delta_{t} w^{n}\right\|^{2}\right\} \\
& +\frac{1}{\Delta t}\left\{\left\|\nabla w^{n+1}\right\|^{2}-\left\|\nabla w^{n}\right\|^{2}+\triangle t^{2}\left\|\nabla \delta_{t} w^{n+1}\right\|^{2}\right\}+\triangle t\left\{\left\|\nabla p^{n+1}\right\|^{2}-\left\|\nabla p^{n}\right\|^{2}\right\}=0,
\end{aligned}
$$

which implies the desired result.

Due to the artificial Dirichlet boundary condition for the pressure, $\left.p^{n+1}\right|_{\Gamma_{c}}=\left.p^{n}\right|_{\Gamma_{c}}=$ $\cdots=0$, the pressure approximation has a poor accuracy which can not be improved by using a higher-order discretization. In fact, it is shown in [11] that the $L^{2}$-error of the pressure approximation converges at the rate of $\frac{1}{2}$. Hence, in order to construct an improved scheme, one needs to resort to the rotational pressure-correction (cf. [11]).

\subsection{Rotational Pressure-Correction Scheme}

The main difference between the standard and rotational pressure-correction schemes is in the computation of the pressure update. The first step is still the same:

Step 1: Given $\left(u^{n}, v^{n}, w^{n}, p^{n}\right)$, compute $\tilde{u}^{n+1} \in \mathbf{H}_{0, \Gamma_{f}}^{1}\left(\Omega_{f}\right)$ and $w^{n+1} \in \mathbf{H}_{0, \Gamma_{s}}^{1}\left(\Omega_{s}\right)$ by solving

$$
\begin{gathered}
\left(\frac{\tilde{u}^{n+1}-u^{n}}{\Delta t}, \varphi\right)_{\Omega_{f}}+\left(\mu \nabla \tilde{u}^{n+1}, \nabla \varphi\right)_{\Omega_{f}}-\left(p^{n}, \operatorname{div} \varphi\right)_{\Omega_{f}}+\left(\frac{\partial w^{n+1}}{\partial \mathbf{n}}, \varphi\right)_{\Gamma_{c}}=0, \\
\tilde{u}^{n+1}=\frac{w^{n+1}-w^{n}}{\Delta t}, \text { on } \Gamma_{c}, \\
\left(\frac{w^{n+1}-2 w^{n}+w^{n-1}}{\Delta t^{2}}, \psi\right)_{\Omega_{s}}+\left(\nabla w^{n+1}, \nabla \psi\right)_{\Omega_{s}}-\left(\frac{\partial w^{n+1}}{\partial \mathbf{n}}, \psi\right)_{\Gamma_{c}}=0
\end{gathered}
$$

for all $\varphi \in \mathbf{H}_{0, \Gamma_{f}}^{1}\left(\Omega_{f}\right)$ and $\psi \in \mathbf{H}_{0, \Gamma_{s}}^{1}\left(\Omega_{s}\right)$.

In the second step, we modify (3.3) as follows:

Step 2: Compute $u^{n+1} \in \mathbf{H}^{1}\left(\Omega_{f}\right)$ and $p^{n+1} \in H^{1}\left(\Omega_{f}\right)$ by solving

$$
\begin{aligned}
& \frac{\left(u^{n+1}-\tilde{u}^{n+1}\right)}{\Delta t}+\nabla\left(p^{n+1}-p^{n}+\lambda \mu \operatorname{div} \tilde{u}^{n+1}\right)=0, \text { in } \Omega_{f}, \\
& \operatorname{div} u^{n+1}=0, \quad \text { in } \Omega_{f}, \\
&\left.u^{n+1} \cdot \mathbf{n}\right|_{\Gamma_{f}}=0 \text { and }\left.p^{n+1}\right|_{\Gamma_{c}}=\left.\left(p^{n}-\lambda \mu \operatorname{div} \tilde{u}^{n+1}\right)\right|_{\Gamma_{c}}
\end{aligned}
$$

where $\lambda \in\left(0, \frac{2}{d}\right)$ (with $d$ being the space dimension) is a preselected parameter. We note that when $\lambda=0$, the scheme is reduced to the standard pressure-correction scheme.

We observe that, thanks to the additional term $\lambda \mu \operatorname{div} \tilde{u}^{n+1}$ in (3.8a), we have an improved pressure B.C. $\left.p^{n+1}\right|_{\Gamma_{c}}=\left.\left(p^{n}-\lambda \mu \operatorname{div} \tilde{u}^{n+1}\right)\right|_{\Gamma_{c}}$. On the other hand, the numerical procedure for the two schemes are essentially identical. 
Theorem 3.2. The scheme (3.7)-(3.8), with $\left.p^{0}\right|_{\Gamma_{c}}=0$, is unconditionally stable. More precisely, if we define the discrete energy as

$$
E^{n+1}=\left\|\delta_{t} u^{n+1}\right\|^{2}+\frac{1}{\Delta t^{2}}\left\|\delta_{t}\left(w^{n+1}-w^{n}\right)\right\|^{2}+\left\|\nabla \delta_{t} w^{n+1}\right\|^{2}+\left\|\nabla q^{n+1}\right\|^{2}+\frac{\lambda \mu}{\triangle t}\left\|\operatorname{div} \tilde{u}^{n+1}\right\|^{2},
$$

then we have

$$
\begin{aligned}
E^{n+1}-E^{n} & +\left\|\delta_{t}\left(\tilde{u}^{n+1}-u^{n}\right)\right\|^{2}+\left\|\delta_{t}\left(w^{n+1}-2 w^{n}+w^{n-1}\right)\right\|^{2} \\
& +\Delta t^{2}\left\|\nabla \delta_{t}\left(w^{n+1}-w^{n}\right)\right\|^{2}+(2-d \lambda) \triangle t \mu\left\|\nabla \tilde{u}^{n+1}\right\|^{2} \leq 0 .
\end{aligned}
$$

Proof. Taking the $\delta_{t}$ operator on system (3.7), we have

$$
\begin{aligned}
& \quad\left(\frac{\delta_{t} \tilde{u}^{n+1}-\delta_{t} u^{n}}{\Delta t}, \varphi\right)_{\Omega_{f}}+\left(\mu \nabla \delta_{t} \tilde{u}^{n+1}, \nabla \varphi\right)_{\Omega_{f}}-\left(\delta_{t} p^{n}, \operatorname{div} \varphi\right)_{\Omega_{f}}+\left(\frac{\partial \delta_{t} w^{n+1}}{\partial \mathbf{n}}, \varphi\right)_{\Gamma_{c}}=0, \\
& \delta_{t} \tilde{u}^{n+1}=\frac{\delta_{t} w^{n+1}-\delta_{t} w^{n}}{\triangle t}, \text { on } \Gamma_{c}, \\
& \left(\frac{\delta_{t} w^{n+1}-2 \delta_{t} w^{n}+\delta_{t} w^{n-1}}{\triangle t^{2}}, \psi\right)_{\Omega_{s}}+\left(\nabla \delta_{t} w^{n+1}, \nabla \psi\right)_{\Omega_{s}}-\left(\frac{\partial \delta_{t} w^{n+1}}{\partial \mathbf{n}}, \psi\right)_{\Gamma_{c}}=0 .
\end{aligned}
$$

Setting $\varphi=2 \triangle t \delta_{t} \tilde{u}^{n+1}$ in (3.9a) and $\psi=2\left(\delta_{t} w^{n+1}-\delta_{t} w^{n}\right)$ in (3.9c), and summing up the two resulting equalities, we have

$$
\begin{aligned}
0= & \left\|\delta_{t} \tilde{u}^{n+1}\right\|^{2}-\left\|\delta_{t} u^{n}\right\|^{2}+\left\|\delta_{t}\left(\tilde{u}^{n+1}-u^{n}\right)\right\|^{2}+2 \mu \Delta t\left\|\nabla \delta_{t} \tilde{u}^{n+1}\right\|^{2} \\
& +\frac{1}{\triangle t^{2}}\left\{\left\|\delta_{t}\left(w^{n+1}-w^{n}\right)\right\|^{2}-\left\|\delta_{t}\left(w^{n}-w^{n-1}\right)\right\|^{2}+\left\|\delta_{t}\left(w^{n+1}-2 w^{n}+w^{n-1}\right)\right\|^{2}\right\} \\
& +\left\|\nabla \delta_{t} w^{n+1}\right\|^{2}-\left\|\nabla \delta_{t} w^{n}\right\|^{2}+\left\|\nabla \delta_{t}\left(w^{n+1}-w^{n}\right)\right\|^{2}-2 \triangle t\left(\delta_{t} p^{n}, \operatorname{div} \delta_{t} \tilde{u}^{n+1}\right)_{\Omega_{f}} .
\end{aligned}
$$

Next we shall use equation (3.8) to deal with the term $2 \triangle t\left(\delta_{t} p^{n} \text {, } \operatorname{div} \delta_{t} \tilde{u}^{n+1}\right)_{\Omega_{f}}$.

Taking $\delta_{t}$ operator on system (3.8), we have

$$
\begin{gathered}
\frac{\delta_{t} u^{n+1}-\delta_{t} \tilde{u}^{n+1}}{\Delta t}+\nabla \delta_{t} q^{n+1}=0, \quad \text { in } \Omega_{f}, \\
\operatorname{div} \delta_{t} u^{n+1}=0, \quad \text { in } \Omega_{f}, \\
\left.\delta u^{n+1} \cdot \mathbf{n}\right|_{\Gamma_{f}}=0 \text { and }\left.q^{n+1}\right|_{\Gamma_{c}}=0,
\end{gathered}
$$

where $q^{n}=p^{n+1}-p^{n}+\lambda \mu \operatorname{div} \tilde{u}^{n+1}$.

Rewrite (3.11a) as

$$
\delta_{t} u^{n+1}+\nabla q^{n+1}=\delta_{t} \tilde{u}^{n+1}+\nabla q^{n}, \quad \text { in } \quad \Omega_{f} .
$$

Taking the inner product with itself from both sides and integrating by parts, thanks to $\left.q^{k}\right|_{\Gamma_{c}}=0$ for all $k$ (due to $\left.q^{0}\right|_{\Gamma_{c}}=0$ ), and $\left.\delta_{t} \tilde{u}^{n+1} \cdot \mathbf{n}\right|_{\Gamma_{f}}=0=\left.\delta_{t} u^{n+1} \cdot \mathbf{n}\right|_{\Gamma_{f}}$, we obtain

$$
\left\|\delta_{t} u^{n+1}\right\|^{2}+\left\|\nabla q^{n+1}\right\|^{2}=\left\|\delta_{t} \tilde{u}^{n+1}\right\|^{2}+\left\|\nabla q^{n}\right\|^{2}-2\left(\operatorname{div} \delta_{t} \tilde{u}^{n+1}, q^{n}\right)_{\Omega_{f}} .
$$


Since $q^{n}=\triangle t \delta_{t} p^{n}+\lambda \mu \operatorname{div} \tilde{u}^{n}$, we have

$$
\begin{aligned}
& -2\left(\operatorname{div} \delta_{t} \tilde{u}^{n+1}, q^{n}\right)_{\Omega_{f}} \\
= & -2\left(\operatorname{div} \delta_{t} \tilde{u}^{n+1}, \Delta t \delta_{t} p^{n}+\lambda \mu \operatorname{div} \tilde{u}^{n}\right)_{\Omega_{f}} \\
= & -2 \triangle t\left(\operatorname{div} \delta_{t} \tilde{u}^{n+1}, \delta_{t} p^{n}\right)_{\Omega_{f}}+\frac{\lambda \mu}{\triangle t}\left(\operatorname{div}\left(\tilde{u}^{n}-\tilde{u}^{n+1}\right), 2 \operatorname{div} \tilde{u}^{n}\right)_{\Omega_{f}} \\
= & -2 \triangle t\left(\operatorname{div} \delta_{t} \tilde{u}^{n+1}, \delta_{t} p^{n}\right)_{\Omega_{f}}+\frac{\lambda \mu}{\triangle t}\left(\left\|\operatorname{div} \tilde{u}^{n}\right\|^{2}-\left\|\operatorname{div} \tilde{u}^{n+1}\right\|^{2}\right)+\lambda \mu \Delta t\left\|\operatorname{div} \delta_{t} \tilde{u}^{n+1}\right\|^{2} .
\end{aligned}
$$

Now using the well-known inequality $\left\|\operatorname{div} \tilde{u}^{n+1}\right\|^{2} \leq d\left\|\nabla \tilde{u}^{n+1}\right\|^{2}$ (with $d=2$ or 3 being the space dimension), and summing up (3.10) (3.12) and (3.13), we obtain

$$
\begin{aligned}
& E^{n+1}-E^{n}+\left\{\left\|\delta_{t}\left(\tilde{u}^{n+1}-u^{n}\right)\right\|^{2}+\frac{1}{\triangle t^{2}}\left\|\delta_{t}\left(w^{n+1}-2 w^{n}+w^{n-1}\right)\right\|^{2}+\left\|\nabla \delta_{t}\left(w^{n+1}-w^{n}\right)\right\|^{2}\right\} \\
\leq & -\left\{2 \mu \triangle t\left\|\nabla \delta_{t} \tilde{u}^{n+1}\right\|^{2}-\lambda \mu \triangle t\left\|\operatorname{div} \delta_{t} \tilde{u}^{n+1}\right\|^{2}\right\} \\
\leq & -(2-\lambda d) \mu \triangle t\left\|\nabla \delta_{t} \tilde{u}^{n+1}\right\|^{2}
\end{aligned}
$$

which implies the desired result.

\subsection{Extension to a nonlinear FSI problem}

The above schemes can be easily extended to deal with the following nonlinear FSI problem [17]:

$$
\begin{array}{ll}
u_{t}-\mu \Delta u+(u \cdot \nabla) u+\nabla p=0, & \text { in } \Omega_{f} \times(0, T), \\
\operatorname{div} u=0, & \text { in } \Omega_{f} \times(0, T), \\
w_{t t}-\Delta w=0, & \text { in } \Omega_{s} \times(0, T)
\end{array}
$$

with the boundary condition:

$$
\begin{array}{ll}
u=0, & \text { on } \Gamma_{f} \times(0, T), \\
w=0, & \text { on } \Gamma_{s} \times(0, T), \\
u=w_{t}, & \text { on } \Gamma_{c} \times(0, T), \\
\frac{\partial w}{\partial \mathbf{n}}=\mu \frac{\partial u}{\partial \mathbf{n}}-p \mathbf{n}-\frac{1}{2}(u \cdot \mathbf{n}) u, & \text { on } \Gamma_{c} \times(0, T) .
\end{array}
$$

For example, a rotational pressure-correction scheme for the above system is:

Step 1: Given $\left(u^{n}, v^{n}, w^{n}, p^{n}\right)$, compute $\tilde{u}^{n+1} \in \mathbf{H}_{0, \Gamma_{f}}^{1}\left(\Omega_{f}\right)$ and $w^{n+1} \in \mathbf{H}_{0, \Gamma_{s}}^{1}\left(\Omega_{s}\right)$ by 
solving

$$
\begin{aligned}
& \left(\frac{\tilde{u}^{n+1}-u^{n}}{\Delta t}, \varphi\right)_{\Omega_{f}}+\left(\mu \nabla \tilde{u}^{n+1}, \nabla \varphi\right)_{\Omega_{f}}+\left(\left(u^{n} \cdot \nabla\right) \tilde{u}^{n+1}, \varphi\right)_{\Omega_{f}} \\
& -\left(p^{n}, \operatorname{div} \varphi\right)_{\Omega_{f}}+\left(\frac{\partial w^{n+1}}{\partial \mathbf{n}}+\frac{1}{2}\left(u^{n} \cdot \mathbf{n}\right) \tilde{u}^{n+1}, \varphi\right)_{\Gamma_{c}}=0, \quad \forall \varphi \in \mathbf{H}_{0, \Gamma_{f}}^{1}\left(\Omega_{f}\right), \\
& \tilde{u}^{n+1}=\frac{w^{n+1}-w^{n}}{\Delta t}, \quad \text { on } \quad \Gamma_{c} \text {, } \\
& \left(\frac{w^{n+1}-2 w^{n}+w^{n-1}}{\triangle t^{2}}, \psi\right)_{\Omega_{s}}+\left(\nabla w^{n+1}, \nabla \psi\right)_{\Omega_{s}}-\left(\frac{\partial w^{n+1}}{\partial \mathbf{n}}, \psi\right)_{\Gamma_{c}}=0, \quad \forall \psi \in \mathbf{H}_{0, \Gamma_{s}}^{1}\left(\Omega_{s}\right) \text {. }
\end{aligned}
$$

Step 2: Compute $u^{n+1} \in \mathbf{H}^{1}\left(\Omega_{f}\right)$ and $p^{n+1} \in H^{1}\left(\Omega_{f}\right)$ by solving

$$
\begin{aligned}
& \frac{\left(u^{n+1}-\tilde{u}^{n+1}\right)}{\Delta t}+\nabla\left(p^{n+1}-p^{n}+\lambda \mu \operatorname{div} \tilde{u}^{n+1}\right)=0, \text { in } \Omega_{f}, \\
& \operatorname{div} u^{n+1}=0, \quad \text { in } \Omega_{f}, \\
& \left.u^{n+1} \cdot \mathbf{n}\right|_{\Gamma_{f}}=0 \text { and }\left.p^{n+1}\right|_{\Gamma_{c}}=\left.\left(p^{n}-\lambda \mu \operatorname{div} \tilde{u}^{n+1}\right)\right|_{\Gamma_{c}}
\end{aligned}
$$

where $\lambda \in\left(0, \frac{2}{d}\right)$ (with $d$ being the space dimension) is a preselected parameter. We note that (3.16a) implies the following weakly satisfied boundary condition

$$
\left.\frac{\partial w^{n+1}}{\partial \mathbf{n}}\right|_{\Gamma_{c}}=\left.\left(\mu \frac{\partial \tilde{u}^{n+1}}{\partial \mathbf{n}}-p^{n} \mathbf{n}-\frac{1}{2}\left(u^{n} \cdot \mathbf{n}\right) \tilde{u}^{n+1}\right)\right|_{\Gamma_{c}}
$$

which is a consistent approximation to (3.15d).

The above scheme is numerically as efficient as its counterpart for the linear FSI problem. However, the stability proof of Theorem 3.2 can not be easily extended to the nonlinear case, since an essential step in the proof is to take the discrete time derivative which leads to complicated nonlinear terms that can not be easily controlled.

\section{A Fourier-Galerkin Spatial Discretization for the case of periodic channel}

As an example, we consider a two-dimensional periodic channel with $\Omega_{f}=(0,2 \pi) \times$ $(0,1), \Omega_{s}=(0,2 \pi) \times(-1,0)$, so $\Omega=(0,2 \pi) \times(-1,1), \Gamma_{f}=\{(x, y) \mid x \in(0,2 \pi), y=1\}$, $\Gamma_{c}=\{(x, y) \mid x \in(0,2 \pi), y=0\}$ and $\Gamma_{s}=\{(x, y) \mid x \in(0,2 \pi), y=-1\}$. We denote $\mathbf{I}^{+}, \mathbf{I}^{-}, \mathbf{I}$ by $\mathbf{I}^{+}=[0,1], \mathbf{I}^{-}=[-1,0]$ and $\mathbf{I}=[-1,1]$. We assume that all functions are periodic in the $x$-direction.

Let $h=(M, N)$ where $M$ is the number of equally spaced points in the $x$-direction, and $N+1$ is the number of Legendre-Gauss-Lobatto points in the $y$ direction of $\Omega_{f}$ and $\Omega_{s}$. For simplicity, we have assumed to use the same number of points in the $y$ direction of $\Omega_{f}$ and $\Omega_{s}$, while in practice, different number of points can be used. Let $P_{N}$ be the set of all 
polynomials of degree less than or equal to $N$. We set

$$
\begin{aligned}
& X_{h}=\left\{v_{h}=\sum_{k=-M / 2}^{M / 2} v_{k}(y) e^{i k x} \text { with } v_{k}(\cdot) \in P_{N}, v_{k}(1)=0\right\}, \mathbf{X}_{h}=X_{h} \times X_{h}, \\
& W_{h}=\left\{w_{h}=\sum_{k=-M / 2}^{M / 2} w_{k}(y) e^{i k x} \text { with } w_{k}(\cdot) \in P_{N}, w_{k}(-1)=0\right\}, \mathbf{W}_{h}=W_{h} \times W_{h}, \\
& M_{h}=\left\{q_{h}=\sum_{k=-M / 2}^{M / 2} q_{k}(y) e^{i k x} \text { with } q_{k}(\cdot) \in P_{N-1}\right\}, \\
& M_{h}^{0}=\left\{q_{h} \in M_{h}:\left.q_{h}\right|_{y=0}=0\right\}, \mathbf{Y}_{h}=\mathbf{X}_{h}+\nabla M_{h}^{0}, \\
& X_{N}^{0}=\left\{v \in H^{1}(I):\left.v\right|_{\mathbf{I}^{+}},\left.v\right|_{\mathbf{I}^{-}} \in P_{N}, v(-1)=v(1)=0\right\}, \mathbf{X}_{N}^{0}=X_{N}^{0} \times X_{N}^{0} .
\end{aligned}
$$

Then, the Fourier-Legendre-Galerkin approximation of the scheme (3.7)-(3.8) is as follows:

Step 1. Let $\tilde{w}_{h}^{n+1}=\delta_{t} w_{h}^{n+1}$, we look for $\left(u_{h}^{n+1}, \tilde{w}_{h}^{n+1}\right) \in \mathbf{X}_{h} \times \mathbf{W}_{h}$ such that

$$
\begin{aligned}
& \alpha\left(\tilde{u}_{h}^{n+1}, \varphi_{h}\right)_{\Omega_{f}}+\left(\nabla \tilde{u}_{h}^{n+1}, \nabla \varphi_{h}\right)_{\Omega_{f}}+\beta\left(\frac{\partial \tilde{w}_{h}^{n+1}}{\partial \mathbf{n}}, \varphi_{h}\right)_{\Gamma_{c}}=<f_{h}^{n}, \varphi_{h}>_{\Omega_{f}}, \quad \forall \varphi_{h} \in \mathbf{X}_{h}, \\
& \tilde{u}_{h}^{n+1}=\tilde{w}_{h}^{n+1}, \quad \text { on } \Gamma_{c}, \\
& \alpha\left(\tilde{w}_{h}^{n+1}, \psi_{h}\right)_{\Omega s}+\beta\left(\nabla \tilde{w}_{h}^{n+1}, \nabla \psi_{h}\right)_{\Omega_{s}}-\beta\left(\frac{\partial \tilde{w}_{h}^{n+1}}{\partial \mathbf{n}}, \psi_{h}\right)_{\Gamma_{c}}=<g_{h}, \psi_{h}>_{\Omega_{s}}, \quad \forall \psi_{h} \in \mathbf{W}_{h},
\end{aligned}
$$

where $\alpha=\frac{1}{\Delta t}, \beta=\Delta t$, and

$$
<f_{h}^{n}, \varphi_{h}>_{\Omega_{f}}:=\alpha\left(u_{h}^{n}, \varphi_{h}\right)_{\Omega_{f}}+\left(p_{h}^{n}, \operatorname{div} \varphi_{h}\right)_{\Omega_{f}}-\left(\frac{\partial w_{h}^{n}}{\partial \mathbf{n}}, \varphi_{h}\right)_{\Gamma_{c}}
$$

and

$$
<g_{h}^{n}, \psi_{h}>_{\Omega_{s}}:=\alpha\left(\tilde{w}_{h}^{n}, \psi_{h}^{n}\right)_{\Omega_{s}}-\left(\nabla w_{h}^{n}, \nabla \psi_{h}\right)_{\Omega_{s}}+\left(\frac{\partial w_{h}^{n}}{\partial \mathbf{n}}, \psi_{h}\right)_{\Gamma_{c}} .
$$

Step 2. Find $\phi_{h}^{n+1} \in M_{h}^{0}$ such that

$$
\left(\nabla \phi_{h}^{n+1}, \nabla q_{h}\right)_{\Omega_{f}}=\frac{1}{\Delta t}\left(\tilde{u}_{h}^{n+1}, \nabla q_{h}\right)_{\Omega_{f}}, \quad \forall q_{h} \in M_{h}^{0}
$$

and compute $u_{h}^{n+1} \in \mathbf{Y}_{h}$ and $p_{h}^{n+1} \in M_{h}$ by

$$
\begin{aligned}
& u_{h}^{n+1}=\tilde{u}_{h}^{n+1}-\Delta t \nabla \phi_{h}^{n+1}, \\
& p_{h}^{n+1}=p_{h}^{n}+\phi_{h}^{n+1}-\lambda \mu Q_{h} \operatorname{div} \tilde{u}_{h}^{n+1},
\end{aligned}
$$

where $Q_{h}$ is a $L^{2}$-projection operator onto $M_{h}$. 
Define

$$
\begin{gathered}
\hat{u}_{h}^{n+1}(\mathbf{x})= \begin{cases}\tilde{u}_{h}^{n+1}(\mathbf{x}), & \text { if } \mathbf{x} \in \Omega_{f}, \\
\tilde{w}_{h}^{n+1}(\mathbf{x}), & \text { if } \mathbf{x} \in \Omega_{s}\end{cases} \\
\hat{\beta}(\mathbf{x}):= \begin{cases}1, & \text { if } \mathbf{x} \in \Omega_{f}, \\
\beta, & \text { if } \mathbf{x} \in \Omega_{s}\end{cases}
\end{gathered}
$$

and

$$
\mathbb{X}_{h}=\left\{\hat{u}_{h} \in \mathbf{H}^{1}(\Omega):\left.\hat{u}_{h}\right|_{\Omega_{f}} \in \mathbf{X}_{h},\left.\hat{u}_{h}\right|_{\Omega_{s}} \in \mathbf{W}_{h}\right\} .
$$

Then, we can rewrite (4.2) as: Find $\hat{u}_{h}^{n+1} \in \mathbb{X}_{h}$ such that

$$
\alpha\left(\hat{u}_{h}^{n+1}, \phi_{h}\right)+\left(\hat{\beta} \nabla \hat{u}_{h}^{n+1}, \nabla \phi_{h}\right)=<f_{h}^{n}, \phi_{h}>_{\Omega_{f}}+<g_{h}^{n}, \phi_{h}>_{\Omega_{s}}, \quad \forall \phi_{h} \in \mathbb{X}_{h} .
$$

Thus, the equation (4.7) can be viewed as a domain-decomposition (with two-domains) approximation to a linear elliptic problem with discontinuous coefficient $\hat{\beta}$.

Expand all the functions in discrete Fourier series, e.g.,

$$
\left(\hat{u}_{h}^{n+1}, f_{h}^{n}, g_{h}^{n}\right)=\sum_{m=-M / 2}^{M / 2}\left(u_{m}^{n+1}(y), f_{m}^{n}(y), g_{m}^{n}(y)\right) e^{i m x} .
$$

Then the system (4.7) is reduced to: For $m=-M / 2, \cdots, 0,1, \cdots, M / 2$, find $u_{m}^{n+1} \in \mathbf{X}_{N}^{0}$ such that

$$
\left(\alpha_{m} u_{m}^{n+1}, \phi\right)_{\mathbf{I}}+\left(\hat{\beta} \frac{d u_{m}^{n+1}}{d y}, \frac{d \phi}{d y}\right)_{\mathbf{I}}=\left(f_{m}^{n}, \phi\right)_{\mathbf{I}^{+}}+\left(g_{m}^{n}, \phi\right)_{\mathbf{I}^{-}}, \quad \forall \phi \in \mathbf{X}_{N}^{0},
$$

where

$$
\alpha_{m}= \begin{cases}\alpha+m^{2}, & \text { if } y \in \mathbf{I}^{+}, \\ \alpha+\beta m^{2}, & \text { if } y \in \mathbf{I}^{-} .\end{cases}
$$

Next we construct a set of basis functions for $X_{N}^{0}$.

We define, for $i=0,1, \ldots, N-2$,

$$
\begin{gathered}
\hat{\varphi}_{i}(y)= \begin{cases}L_{k}(2 y-1)-L_{k+2}(2 y-1), & \text { if } y \in \mathbf{I}^{+}, \\
0, & \text { if } y \in \mathbf{I}^{-}\end{cases} \\
\hat{\varphi}_{N-1+i}(y)= \begin{cases}0, & \text { if } y \in \mathbf{I}^{+}, \\
L_{k}(1+2 y)-L_{k+2}(1+2 y), & \text { if } y \in \mathbf{I}^{-}\end{cases}
\end{gathered}
$$

and the basis function at the interface is

$$
\hat{\varphi}_{2 N-2}= \begin{cases}1-y, & \text { if } y \in \mathbf{I}^{+}, \\ 1+y, & \text { if } y \in \mathbf{I}^{-}\end{cases}
$$

Then

$$
X_{N}^{0}=\operatorname{span}\left\{\hat{\varphi}_{0}, \hat{\varphi}_{1}, \cdots, \hat{\varphi}_{2 N-2}\right\}
$$


Then, writing

$$
u_{m}^{n+1}(y)=\sum_{k=0}^{2 N-2} \hat{u}_{m, k}^{n+1} \hat{\varphi}_{k}(y), \hat{f}_{m, k}^{n}=\left(f_{m}^{n}, \hat{\varphi}_{k}\right)_{\mathbf{I}^{+}}+\left(g_{m}^{n}, \hat{\varphi}_{k}\right)_{\mathbf{I}^{-}},
$$

and taking $\varphi=\hat{\varphi}_{k}$ in (4.9), we can derive the following linear system:

$$
\left(\alpha\left[\begin{array}{lll}
M_{11} & 0 & m_{13} \\
0 & M_{22} & m_{23} \\
m_{31}^{T} & m_{32}^{T} & m_{33}
\end{array}\right]+\left[\begin{array}{lll}
s_{11} & 0 & s_{13} \\
0 & S_{22} & s_{23} \\
s_{31}^{T} & s_{32}^{T} & s_{33}
\end{array}\right]\right)\left[\begin{array}{l}
\bar{u}_{1} \\
\bar{u}_{2} \\
\bar{u}_{3}
\end{array}\right]=\left[\begin{array}{c}
\bar{f}_{1} \\
\bar{f}_{2} \\
\bar{f}_{3}
\end{array}\right],
$$

where $\bar{u}_{1}=\left(\hat{u}_{m, 0}^{n+1}, \hat{u}_{m, 1}^{n+1}, \cdots, \hat{u}_{m, N-2}^{n+1}\right)^{T}, \bar{u}_{2}=\left(\hat{u}_{m, N-1}^{n+1}, \hat{u}_{m, N}^{n+1}, \cdots, \hat{u}_{m, 2 N-3}^{n+1}\right)^{T}$ and $\bar{u}_{3}=u_{m, 2 N-2}^{n+1}$, similarly for $\bar{f}_{1}, \bar{f}_{2}$ and $\bar{f}_{3} ; M_{i j}$ and $S_{i j}$ are block mass and stiffness matrices. We recall that $M_{i i}(i=1,2)$ are penta-diagonal and $S_{i i}(i=1,2)$ are diagonal (cf. $[13,21]$ ). So the linear system can be easily solved by the Schur-complement approach. More precisely, solve first $\bar{u}_{3}$ using a block Gaussian elimination, and then solve $\bar{u}_{1}$ and $\bar{u}_{2}$ separately.

It is clear that (4.5) will be reduced to a sequence of one-dimensional problems in $\mathbf{I}^{+}$ which can be easily solved by a Legendre-spectral method [21].

\section{Numerical Results}

To examine the correctness and accuracy of the proposed numerical schemes, we consider the following non-homogeneous problem

$$
\begin{array}{ll}
u_{t}-\Delta u+\nabla p=f, & \text { in } \Omega_{f} \times(0, T), \\
\operatorname{div} u=0, & \text { in } \Omega_{f} \times(0, T), \\
w_{t t}-\Delta w=g, & \text { in } \Omega_{s} \times(0, T)
\end{array}
$$

with the boundary condition:

$$
\begin{array}{ll}
u=0, & \text { on } \Gamma_{f} \times(0, T), \\
w=0, & \text { on } \Gamma_{s} \times(0, T), \\
u=w_{t}, & \text { on } \Gamma_{c} \times(0, T), \\
\frac{\partial w}{\partial n}=\frac{\partial u}{\partial n}-p \mathbf{n}+h, & \text { on } \Gamma_{c} \times(0, T)
\end{array}
$$

where $\Omega_{f}=(0,2 \pi) \times(0,1), \Omega_{s}=(0,2 \pi) \times(-1,0)$ with periodic boundary conditions in the $x$-direction.

We set the exact solution to be

$$
\begin{aligned}
& u=(-\sin (\pi t) \cos (x) \sin (y-1), \sin (\pi t) \sin (x)(\cos (y-1)-1)), \\
& p=\sin (\pi t) \cos (x) \cos (y), \\
& w=(-\cos (\pi t) \cos (x) \sin (y-1),-\cos (\pi t) \sin (x)(\cos (y+1)-1)) .
\end{aligned}
$$




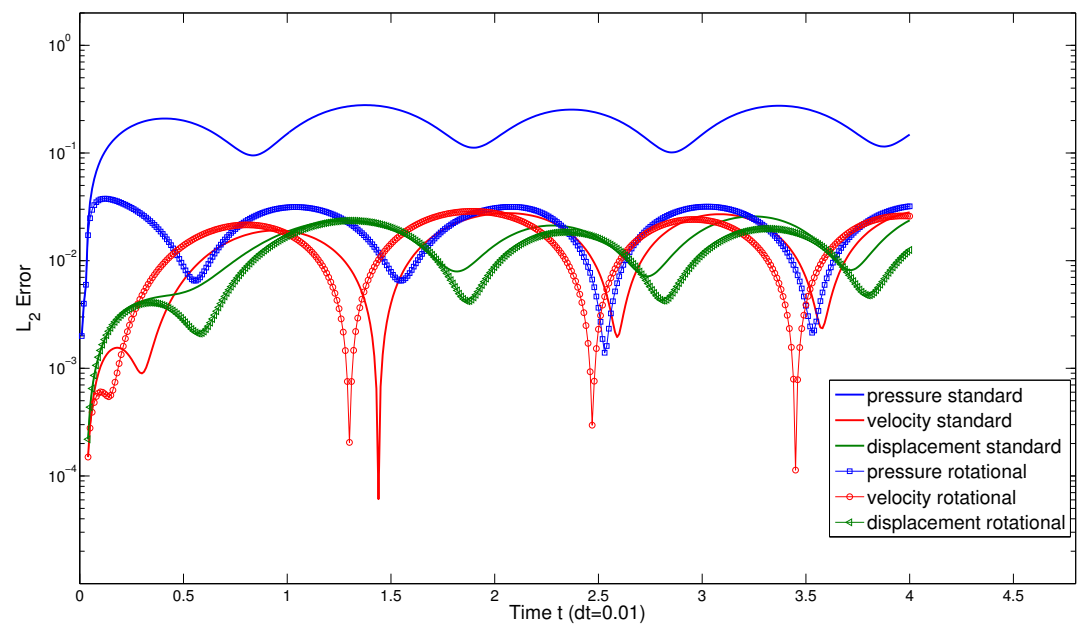

Figure 2: $L^{2}$-errors of first order standard and rotational schemes.

The functions $f, g, h$ can then be computed accordingly.

We employ the Fourier-Legendre method presented in the last section, and choose $(M, N)$ large enough so that the errors are dominated by that from the time discretization. In the following examples, we choose $\lambda=\frac{1}{4}$, which is a preselected parameter introduced in (3.8a).

In Figure 2, we plot evolutions of the $L^{2}$-errors for the pressure, velocity and displacement with the first-order standard and rotational pressure-correction schemes. We observe that the rotational scheme provides much better pressure approximation than the standard scheme.

In Figure 3 and 4, we examine the convergence rates for the first-order standard and rotational schemes. We consider ending time $T=2$ and vary the step size $\Delta t$. In Figure 3 , we observe that the (maximum in time) $L^{2}$ errors for the fluid velocity and the structure displacement in the standard scheme all converge at a rate close to 1 , but the pressure error converges at a rate close to $\frac{1}{2}$. In Figure 4, the convergence rate of the fluid pressure has been improved from order $\frac{1}{2}$ to almost order 1 in the rotational scheme, and the fluid velocity and the structure displacement still converge at a rate close to 1 . These results are consistent with the error estimates for the velocity and pressure given in [11] for Stokes equations with open boundary.

Next, we examine the energy stability of our schemes by solving the homogeneous (with $f, g$ and $h$ being zero) FSI problem with the same initial conditions as in the last example. We compute the discrete energy defined in (3.1) and (3.2), and plot in Figure 5 the discrete energy for standard and rotational schemes with time step $\Delta t=0.01$. We observe that the discrete energy indeed decays monotonically for both schemes. 


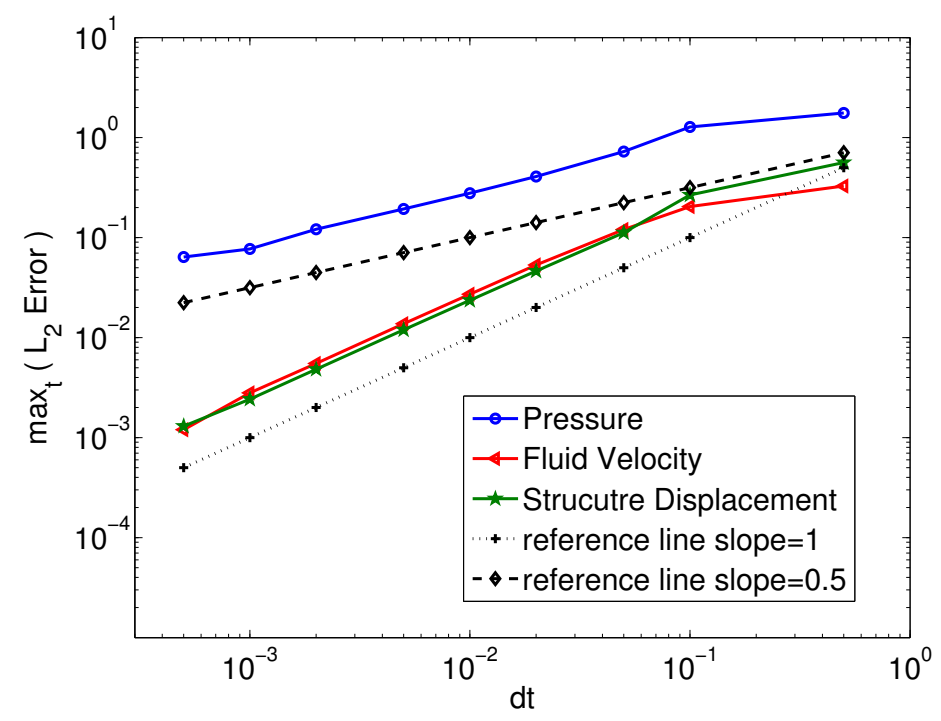

Figure 3: (maximum in time) $L^{2}$-errors of first-order standard scheme.

\section{Conclusion}

We constructed in this paper standard and rotational pressure correction schemes for the linear FSI problem with a fixed interface, and proved rigorously that they are unconditionally energy stable. These schemes are new and fundamental different from the existing schemes. Besides their unconditional stability, they are also computationally very efficient: at each time step, they lead to (i) a coupled linear elliptic system for the velocity and displacement, with the coupling conditions at the interface between the fluid and solid regions, which can be efficiently solved by using a standard domain decomposition (with two domains) approach; and (ii) a discrete Poisson equation in the fluid region.

We validated these schemes by using a Fourier-Legendre spatial discretization for the FSI problem in a periodic channel. In particular, our numerical results indicate that the convergence rates of the first-order rotational scheme for the velocity, pressure and displacement in $L^{2}$-norm are close to order one.

Although we only considered the linear FSI problem with fixed interface, we believe that the essential approaches used here in constructing our numerical schemes can be extended to nonlinear FSI problems and/or with moving interface.

\section{Acknowledgments}

This work is partially supported by NSF DMS-1215066 and AFOSR FA9550-11-1-0328. 


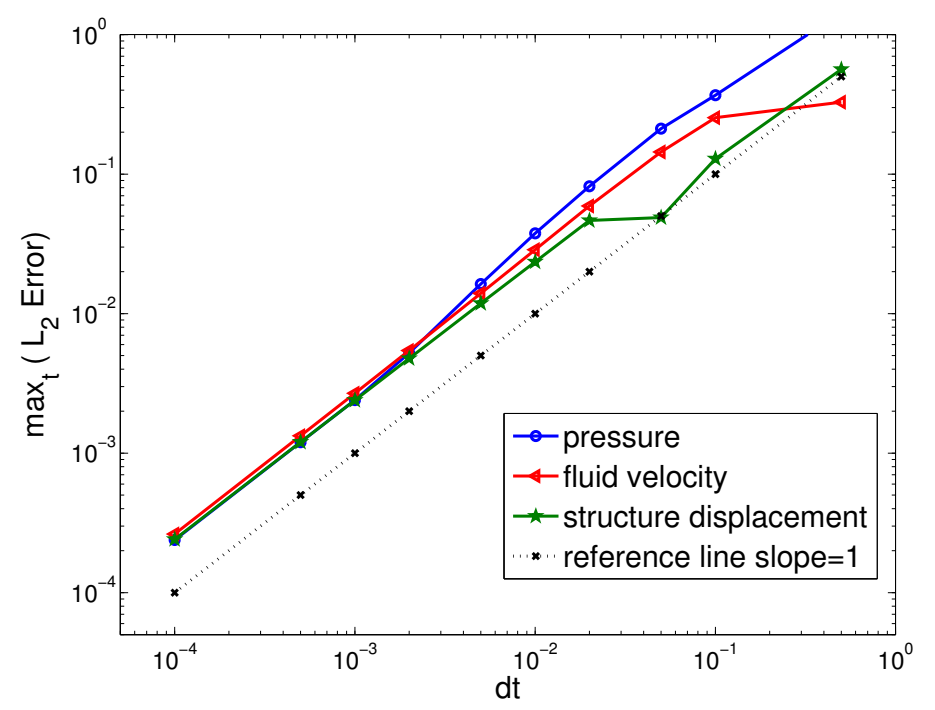

Figure 4: (maximum in time) $L^{2}$-errors of first-order rotational scheme.

\section{References}

[1] S. Badia and R. Codina. On some fluid-structure iterative algorithms using pressure segregation methods. Application to aeroelasticity. Internat. J. Numer. Methods Engrg., 72(1):46-71, 2007.

[2] S. Badia, F. Nobile, and C. Vergara. Fluid-structure partitioned procedures based on Robin transmission conditions. J. Comput. Phys., 227(14):7027-7051, 2008.

[3] S. Badia, A. Quaini, and A. Quarteroni. Splitting methods based on algebraic factorization for fluid-structure interaction. SIAM J. Sci. Comput., 30(4):1778-1805, 2008.

[4] E. Burman and M. A. Fernández. Stabilized explicit coupling for fluid-structure interaction using Nitsche's method. C. R. Math. Acad. Sci. Paris, 345(8):467-472, 2007.

[5] S. K. Chakrabarti, S. Hernandez, and C. A. Brebbia, editors. Fluid structure interaction and moving boundary problems, volume 43 of Advances in Fluid Mechanics. WIT Press, Southampton, 2005. Edited papers from the 3rd International Conference on Fluid Structure Interaction and the 8th International Conference on Computational Modelling and Experimental Measurements of Free and Moving Boundary Problems held in La Coruna, September 19-21, 2005.

[6] Q. Du, M. D. Gunzburger, L. S. Hou, and J. Lee. Semidiscrete finite element approximations of a linear fluid-structure interaction problem. SIAM J. Numer. Anal., 42(1):1-29, 2004.

[7] C. Farhat, M. Lesoinne, and P. LeTallec. Load and motion transfer algorithms for fluid/structure interaction problems with non-matching discrete interfaces: momentum and energy conservation, optimal discretization and application to aeroelasticity. Comput. Methods Appl. Mech. Engrg., 157(1-2):95-114, 1998.

[8] C. A. Felippa, K. Park, and C. Farhat. Partitioned analysis of coupled mechanical systems. 190:3247âĂŞ3270, 2001.

[9] M. A. Fernández. Coupling schemes for incompressible fluid-structure interaction: implicit, semi-implicit and explicit. SëMA J., (55):59-108, 2011.

[10] M. A. Fernández, J.-F. Gerbeau, and C. Grandmont. A projection semi-implicit scheme for 


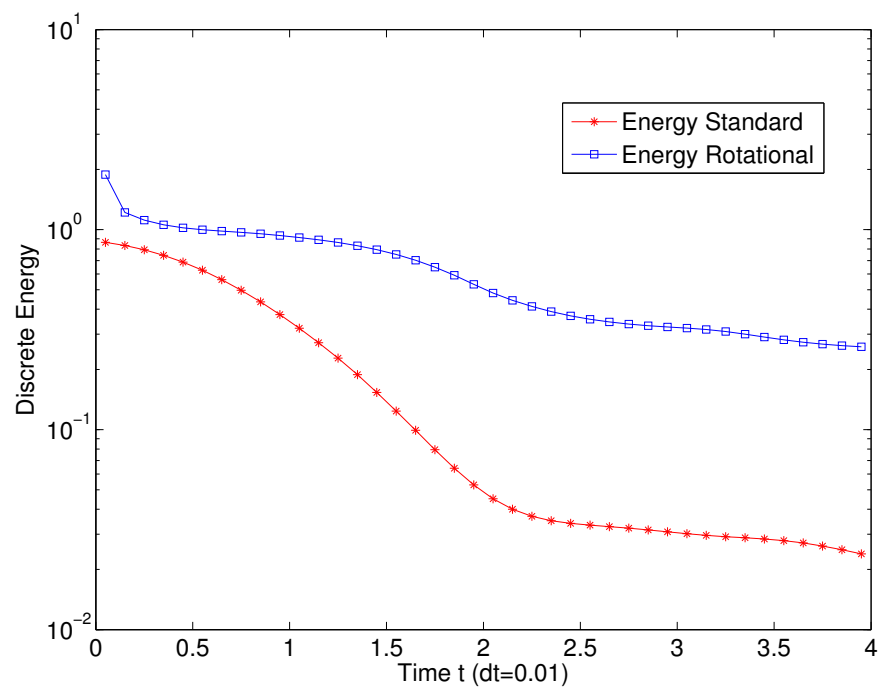

Figure 5: Plots of discrete energy for standard and rotational schemes with time step 0.01 .

the coupling of an elastic structure with an incompressible fluid. Internat. J. Numer. Methods Engrg., 69(4):794-821, 2007.

[11] J. L. Guermond, P. Minev, and J. Shen. Error analysis of pressure-correction schemes for the time-dependent Stokes equations with open boundary conditions. SIAM J. Numer. Anal., 43(1):239-258 (electronic), 2005.

[12] J. L. Guermond, P. Minev, and J. Shen. An overview of projection methods for incompressible flows. Comput. Methods Appl. Mech. Engrg., 195(44-47):6011-6045, 2006.

[13] Y. He, D. P. Nicholls, and J. Shen. An efficient and stable spectral method for electromagnetic scattering from a layered periodic structure. J. Comput. Phys., 231(8):3007-3022, 2012.

[14] G. Hou, J. Wang, and A. Layton. Numerical methods for fluid-structure interaction-a review. Commun. Comput. Phys., 12(2):337-377, 2012.

[15] J. Hron and S. Turek. A monolithic FEM/multigrid solver for an ALE formulation of fluidstructure interaction with applications in biomechanics. Springer, 2006.

[16] B. Hübner, E. Walhorn, and D. Dinkler. A monolithic approach to fluid-structure interaction using space-time finite elements. Computer methods in applied mechanics and engineering, 193(23):2087-2104, 2004.

[17] I. Kukavica, A. Tuffaha, and M. Ziane. Strong solutions to a nonlinear fluid structure interaction system. J. Differential Equations, 247(5):1452-1478, 2009.

[18] U. Küttler and W. A. Wall. Fixed-point fluid-structure interaction solvers with dynamic relaxation. Computational Mechanics, 43(1):61-72, 2008.

[19] H. G. Matthies and J. Steindorf. Partitioned strong coupling algorithms for fluidâĂŞstructure interaction. 81:805-812, 2003.

[20] A. Quarteroni and A. Valli. Domain decomposition methods for partial differential equations. Numerical Mathematics and Scientific Computation. The Clarendon Press Oxford University Press, New York, 1999. Oxford Science Publications.

[21] J. Shen. Efficient spectral-Galerkin method. I. Direct solvers of second- and fourth-order equations using Legendre polynomials. SIAM J. Sci. Comput., 15(6):1489-1505, 1994.

[22] A. Toselli and O. Widlund. Domain decomposition methods-algorithms and theory, volume 34 
of Springer Series in Computational Mathematics. Springer-Verlag, Berlin, 2005. 